\title{
Nonlinear and Robust Internal Model Control of a Piezoelectric Actuator Devoted to Characterization at the Micro/Nanoscale
}

\author{
Micky RaKotondrabe, Omar AlJanaideh, and Mohammad Al JAnaideh
}

\begin{abstract}
In this paper, a nonlinear robust internal model control is suggested to control a piezoelectric actuator that is typified by hysteresis, creep phenomenon and badly damped behavior. The principle of the proposed methodology consists of combining three techniques: i) considering the creep as internal disturbance that can be rejected using a disturbance observer/compensator, ii) eliminating of hysteresis with a feedforward compensator, and iii) handling the dynamics and furnishing the global robustness using a feedback linear internal model controller. The overall nonlinear controller was implemented and verified through hardware-in-the-loop experimental tests to explore its efficiency. The results revealed that hysteresis nonlinearity in excess of $14 \%$ was reduced to less than $1 \%$, creep in excess of $22 \%$ was completely removed, and finally oscillation with overshoot of $35 \%$ was completely damped.
\end{abstract}

\section{INTRODUCTION}

Piezoelectric actuators are considered decent choice for applications that require positioning/manipulating objects at micro/nano scale levels in micro-seconds. Examples of these applications include actuation of proportional-flow control valves [1], transportation of micro objects in sensitive applications [2], and operating of fuel injection systems [3]. However, these actuators exhibit hysteresis and creep nonlinearities that degrade their positioning accuracy [4], [5]. Moreover, the geometric structure of piezoelectric actuators adds linear dynamics to the hysteresis and creep nonlinearities [6]-[14]. Such nonlinearities yield oscillations and instabilities in open and closed-loop control systems [6].

The control of systems that exhibit hysteresis and creep effects necessitates formulating a model that can describe these nonlinearities. Consequently, different methodologies have been suggested to characterize the nonlinear properties of these systems. Hammerstein system-based Prandtl-Ishlinskii model [10], [11], Hammerstein system-based Preisach model [14], [15], and Hammerstein system-based Bouc-wen model [16], [17] are examples of models that have been used to synthesize open-loop and feedback control techniques to stabilize smart material-based actuators. Some other studies have further considered addressing the effects of creep beside the control of hysteresis nonlinearities [18]-[20].

M. Rakotondrabe is with the Department of Automatic Control and Micro-Mechatronic Systems, FEMTO-ST, AS2M, Univ. Bourgogne Franche-Comté, Univ. de Franche-Comt/CNRS/ENSMM/UTBM, FEMTOST Institute, Besançon France, email: mrakotonefemto-st.fr

O. Aljanaideh is Senior Control Engineer at ASML-HMI, San Jose, CA USA. email: omar.al janaideheasml.com.

M. Al Janaideh is with the Department of Mechanical Engineering, Memorial University of Newfoundland, St. John's, Newfoundland A1B 3X5, Canada. email: mal janaideh@mun.ca.
The adequate consideration of piezoelectric actuators to serve different precision motion applications requires proposing a comprehensive control technique that can cancelout the effects of creep, hysteresis, and also dampen the linear dynamics. Consequently, it is essential to formulate a model that can account for all these undesirable properties. This study proposes an internal model control with inverse Prandtl-Ishlinskii model to enhance the tracking performance of piezocantilever actuator that shows hysteresis, creep and high oscillations in real-time system. For modeling hysteresis properties of the actuator, the study considers the PrandtlIshlinskii model so as to formulate an exact inverse that permits obtaining an exact mathematical model for the inverse compensation errors [11].

The paper is organized as follows. Section II describes The experimental setup, the creep-Hammerstein model for the piezocantilever actuator, and the parameters identification. Section III introduces the proposed internal model control to stabilize the piezocantilever actuator considering hysteresis, creep, and oscillations. The experimental results that examine the effectiveness of the proposed internal model control in real-time are presented in Section IV. The conclusions and future research are summarized in Section V.

\section{THE EXPERIMENTAL SETUP AND THE NONLINEAR MODEL}

\section{A. The experimental setup}

The piezoelectric actuator that was used in the experimental study is depicted in Fig.1. As the figure shows the actuator has a cantilever structure with rectangular section. The actuator is composed of several layers of lead-zirconatetitanate (PZT) piezoelectric material and has $25 \mathrm{~mm}$ active length (out of the clamping), $1 \mathrm{~mm}$ width, and $1 \mathrm{~mm}$ total thickness. The actuating signals are applied to the actuator via electrodes that were furnished on the top and bottom surfaces of the actuator. The function of the actuator allows bending along $y$ axis or $z$ axis based on sensitization of input signal $u_{y}$ or $u_{z}$. The output movements/displacements have high resolution (down to tens of nanometer) within high bandwidth (up to several hundreds of Hertz). These advantages make this actuator well-suited for wide range of micro-scale applications such as micromanipulation, precise positioning, micro-scale characterization [4].

Although piezocantilever actuators produce the output displacement with a fine resolution, this nano-level of displacement comes at the cost of hysteresis and creep nonlinearities. These nonlinearities find their origin in the complex properties of piezoelectric ceramics and ferro-electric 
materials limit the implementation of these actuators in the above mentioned applications to a narrow operating range of input amplitudes and frequencies as they could drastically compromise the success of the tasks in these applications. Furthermore, the cantilever structure based piezoactuator exhibits badly damped oscillation which is inherited from the high stiffness of the material combined with the geometrical cantilever structure. This badly damped oscillations have several drawbacks: increase of the settling time (or stabilization time) although the actuator exhibits a very low rise time, degradation of the stability and of the general performances of the tasks. The nonlinearities and the oscillating dynamics have therefore to be controlled in a proper way.

The experimental setup employed in the sequel is composed of the piezoelectric actuator. In this paper, we consider the motion in $y$ axis. A displacement sensor that measures the deflection along $y$ axis considered as output. The sensor is an optical sensor with triangulation principle and from Keyence company (LC2420). This sensor is tuned to have a measurement resolution of $10 \mathrm{~nm}$ and a bandwidth of $5 \mathrm{kHz}$ which is sufficient for the experimental study in the paper. A computer with MATLAB-Simulink software is used for the acquisition of the measurement $y$, the generation of the driving voltage $u=u_{y}$ as well as to implement the controller. Finally, a dSPACE acquisition board serves as interface between the computer and the rest. The sampling frequency of the whole acquisition system is set to $20 \mathrm{kHz}$.

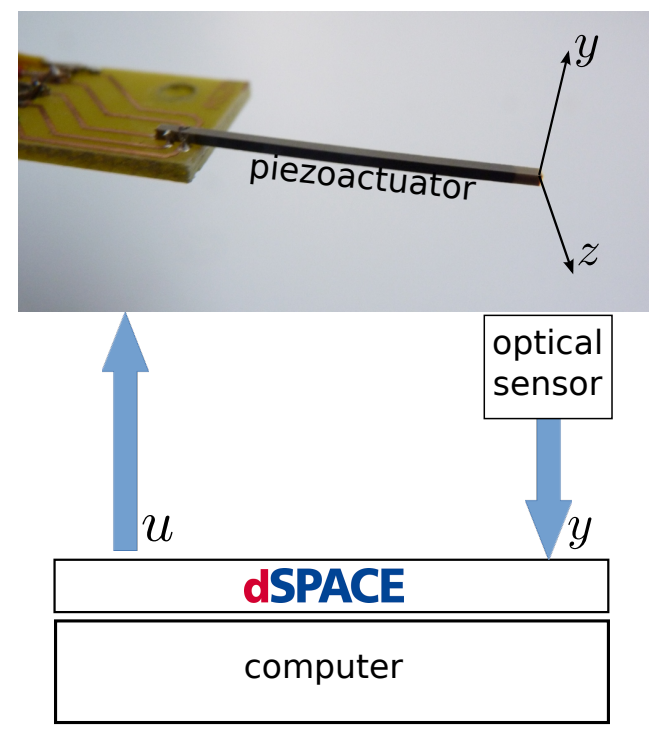

(a)

Fig. 1. (a) The experimental setup with piezocantilever, measured nonlinear dynamics of the piezocantilever: (b) hysteresis nonlinearity, and (c) creep nonlinearity.

\section{B. Creep-Hammerstein model}

In this section, we consider a dynamic structure that simultaneously accounts for the creep, hysteresis, and dynamics of the piezoelectric actuator. This structure is called as creep-Hammerstein model for the piezoelectric actuator. Analytically, the output of the model is expressed as [4], [5]

$$
y(s)=\mathcal{P}[u(s)] D(s)+C_{r}(s) u(s),
$$

where $\mathcal{P}[u(s)]$ is a hysteresis operator that characterizes the hysteresis nonlinearity of the piezocantilever, $D(s)$ is a linear dynamics that describes the mechanical structure behavior, and $C_{r}(s)$ is a linear dynamic that accounts for the creep nonlinearity. Fig.2 depicts the block diagram corresponding to the model in Eq.1. Since the static gain is nonlinear and encompassed in the hysteresis term $\mathcal{P}$, the dynamics $D(s)$ is normalized such that $D(s=0)=1$.

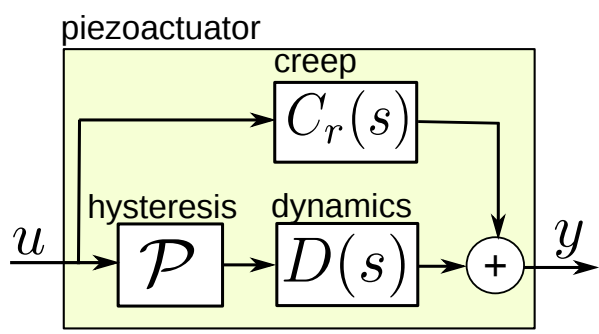

Fig. 2. Creep-Hammerstein dynamic representation for the piezoelectric actuator, where $\mathcal{P}[u(s)]$ is a hysteresis operator, $D(s)$ is a linear dynamics, and $C_{r}(s)$ is a linear dynamics for the creep nonlinearity.

\section{Model parameters identification}

The characterization of the actuator and the identification of the hysteresis model $\hat{\mathcal{P}}$, the creep model $\hat{C}_{r}(s)$ and the dynamics $\hat{D}(s)$ are given in this subsection. Note that $\hat{\mathcal{P}}$, $\hat{C}_{r}(s)$ and $\hat{D}(s)$ are approximate models characterize $\mathcal{P}$, $C_{r}(s)$ and $D(s)$.

1) The hysteresis model: Prandtl-Ishlinskii model is used in this section to describe the hysteresis nonlinearities of the actuator. This model has been employed widely in the literature to represent the hysteresis of smart material-based actuators [11]. The model can characterize the symmetric hysteresis in piezoactuators, and its inverse is exact and can be obtained analytically to compensate for the hysteresis effects in real-time system [21]. The Prandtl-Ishlinskii model $\mathcal{P}$ is a summation of weighted play operators $\mathcal{Q}_{\rho_{j}}$ of thresholds $\rho_{j}$ and input voltage $u(t)$ as

$$
\mathcal{P}(t)=\sum_{j=1}^{J} g_{j} \mathcal{Q}_{\rho_{j}}[u](t),
$$

where $J$ is an integer represents the number of the play operators that can be used to formulate the model, and $g_{j}$ are the weights which govern the slope of the play operators. In this paper, we consider the threshold function $\rho_{j}=\alpha j$ and the weighting function $g_{j}=\beta e^{-\gamma \rho_{j}}$, where $\alpha, \beta$, and $\gamma$ are positive constants. The parameters of the model were identified via MATLAB Optimization Toolbox with least square nonlinear method under the constrains of $\alpha>0, \beta>0$, and $\gamma>0$, and $J=10$. More details about the model and its properties are presented in [11]. 
Fig.3(a) shows comparison between the output of model $\hat{\mathcal{P}}$ and the measured hysteresis nonlinearities of a sinusoidal input voltage of $u$ under $0.1 \mathrm{~Hz}$ excitation frequency and $10 \mathrm{~V}$ amplitude. The percentage of the hysteresis nonlinearity is $\frac{h}{H} \approx \frac{8 \mu m}{2 \times 28 \mu m} \approx 14.3 \%$.

2) linear dynamic model: This section presents identification of the linear dynamics $\hat{D}(s)$. A step driving voltage of $10 \mathrm{~V}$ was applied to excite the piezoactuator. Then, using the measured step response and the ARMAX (AutoregressiveMoving Average with Exogenous) model of MATLAB Systems Identification Toolbox [22], the linear dynamics $\hat{D}(s)$ is obtained. The model $\hat{D}(s)$ is derived by normalizing the identified dynamics such that $\hat{D}(s=0)=1$. Eq. 3 presents the identified model. Fig.3(b) shows comparison between the identified model $\hat{D}(s)$ and the measured displacement step response. The figure shows an overshoot of $35 \%$. The identified model is

$$
\hat{D}(s)=\frac{0.35684(s+4005)\left(s^{2}+5441 s+3.4 e 7\right)}{(s+2197)\left(s^{2}+48.93 s+2.4 e 7\right)} .
$$

3) Creep model: The creep model $\hat{C}_{r}(s)$ is a transfer function that approximates a nonlinear behavior. Such approximation has been established in various applications of piezocantilever actuators. Again, a step voltage $u=10 \mathrm{~V}$ is applied to the piezocantilever actuator. Rather than observing only for tens of milliseconds as for the dynamics, applying the step response over several minutes contributes a slow drift. This drift is the creep nonlinearity. We separate the experimental data corresponding to this drift, the creep nonlinearity, from the whole step response. Using MATLAB Systems Identification Toolbox and the experimental data related to the drift, a linear model is identified. Fig.3(b) depicts the response of the identified model compared to the experimental creep. The results reveals a creep in excess of $\frac{6.3 \mu m}{28 \mu m} \approx 22.5 \%$. The creep model is

$$
\hat{C}_{r}(s)=\frac{3 \times 10^{-4}(s+25)(s+0.01)}{(s+0.12)(s+0.004)} .
$$

The obtained hysteresis, creep, and linear dynamics models Eqs. (2), (3), and (4) are used to formulate creepHammerstein model for the piezocantilever actuator. This model is considered in the next section to propose nonlinear internal- model control that can compensate for the hysteresis, rejects disturbances due to the creep nonlinearity, and stabilize the linear dynamics due to the step input voltage.

\section{ROBUST AND NONLINEAR INTERNAL MODEL CONTROL}

In this section we present the proposed nonlinear internal model control. First, we compensate for hysteresis and creep nonlinearities with the inverse model $\mathcal{P}$ and $\hat{C}(s)$. Then, we demonstrate that we have linear dynamic model $D(s)$ with a fictive disturbance. Considering that the linear model could exhibit uncertainties, we afterwards apply a linear internal model control. This feedback control permits maintaining some prescribed tracking performance despite of the above
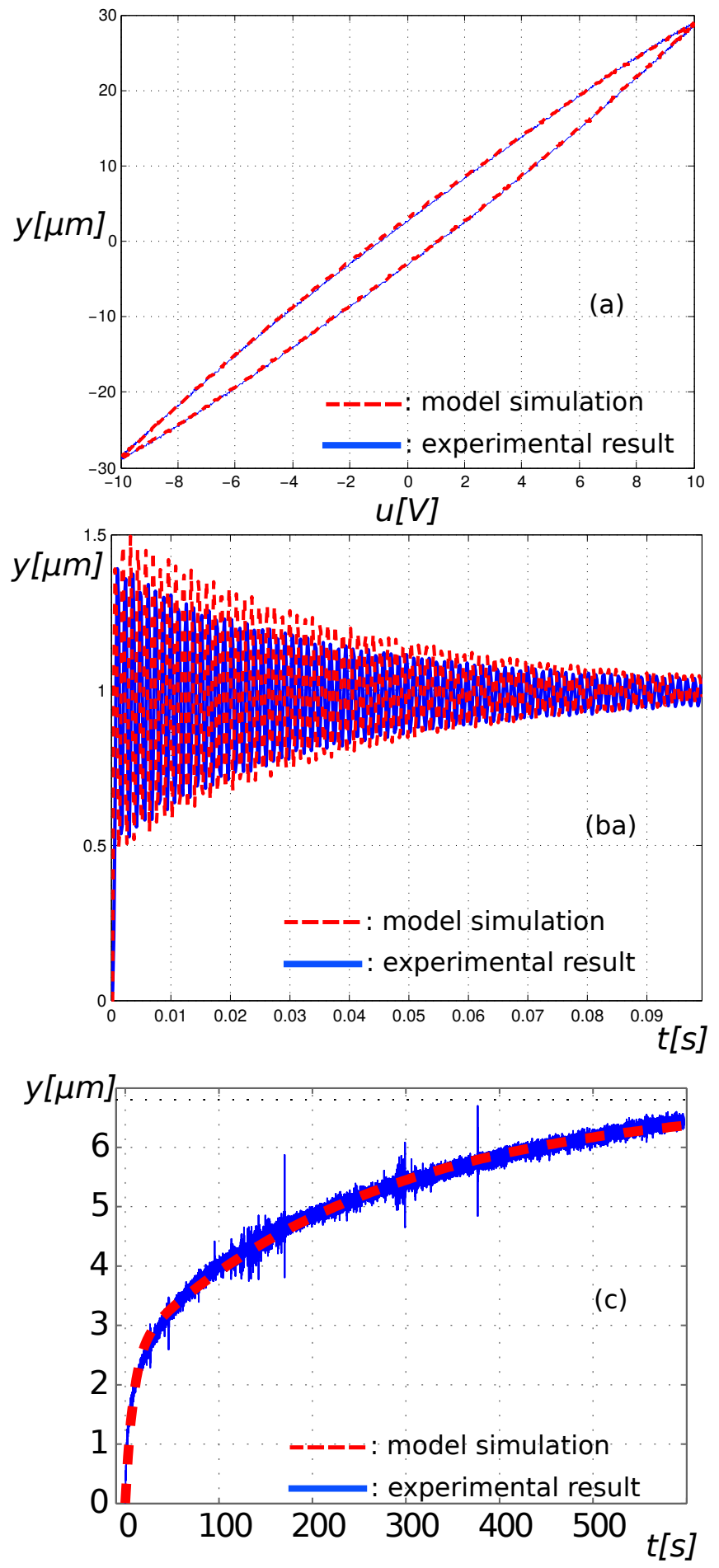

Fig. 3. Characterization and models responses of: (a) the hysteresis, (b) the linear dynamic model, and (c) the creep model.

model uncertainties and the fictive disturbance. The nonlinear and robust internal model controller (NR-IMC) is therefore composed of the nonlinear compensators and the linear internal model controller (L-IMC). 


\section{A. Hysteresis and creep compensation}

In order to compensate for the hysteresis nonlinearities of $\mathcal{P}$, we use the classical cascade compensation approach as illustrated in Fig.4(a) where

$$
\mathcal{P}^{-1}\left[u_{s}\right](t)=\sum_{j=1}^{J} q_{j} \mathcal{Q}_{\hat{z}_{j}}\left[u_{s}\right](t),
$$

where $q_{j}$ are the weights of the inverse, and $z_{j}$ are the thresholds of the inverse model. More details about the inverse model and its properties are presented in [11].

Regarding the creep, since this latter is an additive term according to Eq. 1, we suggest a compensation with subtraction, as shown in Fig.4(a). Since the identified models of the hysteresis and of the creep could slightly differ from the real phenomena and thus represent uncertainties, the compensators are therefore approximate. We denote them as: $\hat{\mathcal{P}}^{-1}\left[u_{s}(s)\right]$ and $\hat{C}_{r}(s)$. Consequently, the output $o(s)$ in Fig.4(a) is expressed as

$$
o(s)=\left(C_{r}(s)-\hat{C}_{r}(s)\right) u(s)+\mathcal{P}\left[\hat{\mathcal{P}}^{-1}\left[u_{s}(s)\right]\right] D(s) .
$$

It is however shown that the compensation of a hysteresis $\mathcal{P}$ with a compensator from an approximate model $\hat{\mathcal{P}}$ provides a linear model with a bounded fictive disturbance [11]. Consequently

$$
\mathcal{P}\left[\hat{\mathcal{P}}^{-1}\left[u_{s}(s)\right]\right] D(s)=D(s) u_{s}(s)+e_{P}(s),
$$

where $e_{P}(s)$ is a bounded disturbance. On the other hand, if the creep compensation was exact, i.e. $\hat{C}_{r}(s)=C_{r}(s)$, we would have $\left(C_{r}(s)-\hat{C}_{r}(s)\right) u(s)=0$. If it is not the case, which in general is the case in real-time systems, we have

$$
\left(C_{r}(s)-\hat{C}_{r}(s)\right) u(s)=e_{C}(s),
$$

where $e_{C}(s)$ is a positioning error signal and can be considered as fictive disturbance. Since $C_{r}(s)$ and $\hat{C}_{r}(s)$ are stable transfer functions [18], [19], [20] and the positioning error signals are $\|u(t)\|_{\infty}<\eta$ and $\left\|e_{C}(t)\right\|_{\infty}<\eta$, where $\eta$ is a positive constant, we conclude with Eq. 6, Eq. 7 and Eq. 8 that

$$
o(s)=D(s) u_{s}(s)+e(s),
$$

where

$$
e(s)=e_{P}(s)+e_{C}(s),
$$

is a bounded fictive disturbance that embraces the uncertainties due to characterization and compensation of hysteresis and creep nonlinearities. Thus, Fig.4(a) is equivalent to Fig.4(b) where the new system to be controlled is a linear model with an output disturbance.

\section{B. Internal model control}

The principle of an internal model control (IMC) consists of incorporating a model of the process in control design [23]. There are several applications of IMC, and one of the most used is the Smith predictor, which is used to

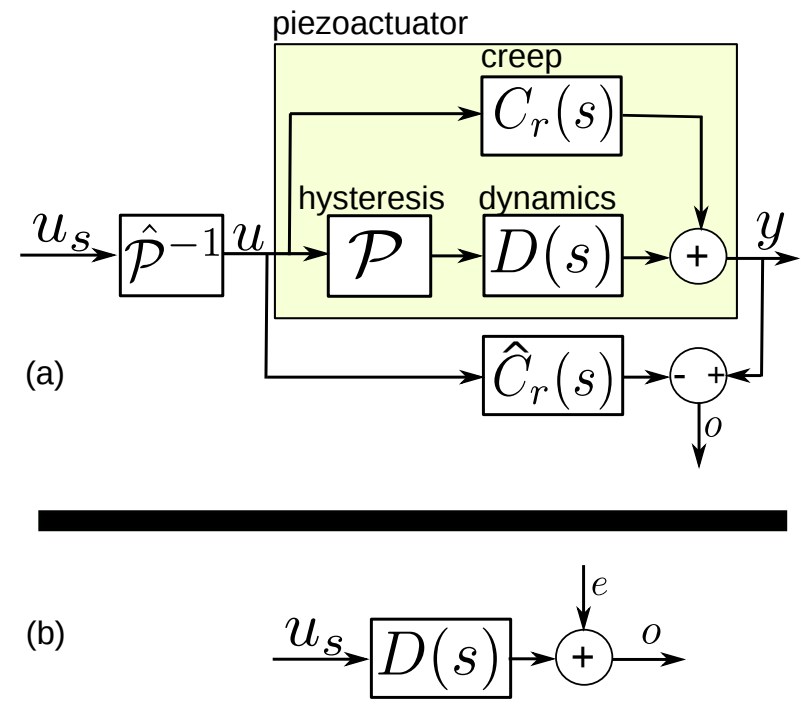

Fig. 4. (a) hysteresis and creep compensation, and (b) equivalent scheme of (a).

control linear systems with delay [24]. For linear systems, IMC is particularly an efficient and simple to implement method to control considering bounded uncertainties and output disturbance. This is the case for the system displayed in Fig.4(b).

Consider the linear IMC feedback control in Fig.5(a). In this, $\hat{D}(s)$ is the approximate model of the dynamics $D(s)$ of the piezocantilever actuator as we identified in Eq. 3, $\kappa(s)$ is the feedback controller to be designed, while $y_{r}(s)$ is the reference displacement input. Considering $\hat{D}(s)$ slightly different from the real dynamics $D(s)$ is worthy. Thus, similar to the cases of hysteresis and creep, the real dynamic behavior is complex. Then, an exact model would be hard to obtain. From Fig.5(a), we have

$$
y=\frac{\kappa D}{(1+\kappa(D-\hat{D}))} y_{r}+\frac{(1-\kappa \hat{D})}{(1+\kappa(D-\hat{D}))} e .
$$

We choose the controller $\kappa(s)$ as

$$
\kappa(s)=\frac{1}{\hat{D}(s)} W(s),
$$

where $W(s)$ is a gabarit, also called filter, that transcripts the specification related to the desired tracking performance. The most simple but meaningful desired specifications for the closed-loop include a small settling time of $t_{r}$, no oscillation (full damping), and no static error. From these specifications, the following gabarit is suggested

$$
W(s)=\frac{1}{\left(1+\frac{t_{r}}{3} s\right)} .
$$

If we have an exact model $\hat{D}(s)=D(s)$, the closed-loop equation in Eq. 11 becomes

$$
y(s)=W(s) y_{r}(s)+(1-W(s)) e(s) .
$$


From Eq. 14, we conclude that the specified tracking performance is satisfied $\left(y(s)=W(s) y_{r}(s)\right)$ and the disturbance is rejected with a complementary dynamics $y(s)=$ $(1-W(s)) e(s)$. At steady-state conditions, the static error is zero and the disturbance is fully rejected as

$$
y(0)=y_{r}(0)+0 \cdot e(0) .
$$

Notice that the obtained performance in Eq. 15 is valid even if the hysteresis and creep models and compensations were not exact. Hence, they are incorporated in the disturbance term $e(s)$ which is rejected.

In real case, however, the employed dynamic model $\hat{D}(s)$ could differ from the real dynamics $D(s)$ and thus we have $\hat{D}(s) \neq D(s)$. Consequently, from Eq. 11 and Eq. 12, the closed-loop equation is $y(s)=$

$$
\frac{\frac{D(s)}{\hat{D}(s)} W(s)}{\left(1+W(s)\left(\frac{D(s)}{\hat{D}(s)}-1\right)\right)} y_{r}(s)+\frac{(1-W(s))}{\left(1+W(s)\left(\frac{D(s)}{\hat{D}(s)}-1\right)\right)} e(s)
$$

Because $D(s)$ and $\hat{D}(s)$ are normalized dynamics, at steady-state condition, Eq. 16 comes back to Eq. 15 and the tracking performance as well as the disturbance rejection are satisfied. However, the transient part to reach the reference and the transient part to reject the disturbance could slightly differ from the specifications (in terms of settling time and overshoot) depending on the deviation between $\hat{D}(s)$ and $D(s)$, which is quantified by $\frac{D(s)}{\hat{D}(s)}$.

We also note from Eq. 12 that the suggested controller $\kappa(s)$ requires an inverse of the dynamic model $\hat{D}(s)$. Dynamics inversion is often a tricky task because it requires an invertible model, i.e. $\hat{D}(s)$ should be minimum phase (with stable zero) and bi-causal (its inverse is also causal). Consequently, two possibilities are considered. If the identified model $\hat{D}(s)$ is invertible, the controller is taken to be that of Eq. 12. If $\hat{D}(s)$ is not invertible, the following controller can be applied

$$
\kappa(s)=\frac{1}{\hat{D}(0)} W(s)=W(s) .
$$

With this latter controller, the closed-loop equation in (11) becomes $y(s)=$

$$
\frac{D(s) W(s)}{(1+W(s)(D(s)-1))} y_{r}(s)+\frac{(1-W(s))}{(1+W(s)(D(s)-1))} e(s) .
$$

Then, the tracking performance specification and the disturbance rejection at steady-state condition in Eq. 15 are always satisfied. Regarding the transient parts, this case has more deviation than that of Eq. 16, the perfect transient parts being that expressed in Eq. 14.

\section{EXPERIMENTAL RESULTS}

While the calculation of the previous section dealt with the calculation of a linear IMC controller, as displayed in Fig. 5(a), the final controller to be implemented and applied to the piezoactuator is a nonlinear IMC. Indeed, it is composed of:
- the hysteresis compensator $\hat{\mathcal{P}}^{-1}$ which is nonlinear,

- the creep compensator $\hat{C}_{r}(s)$,

- the dynamics $\hat{D}(s)$,

- and the feedback controller $\kappa(s)=\frac{1}{\hat{D}(s)} W(s)$ or $\kappa(s)=$ $W(s)$ according if $\hat{D}(s)$ is invertible or not.

As we demonstrated in the precedent section, the whole controller is robust in the sense that steady-state tracking performances and disturbance rejection are always satisfied, despite of the deviation between the used models $\hat{\mathcal{P}}, \hat{C}_{r}(s)$ and $\hat{D}(s)$ from the real behavior $\mathcal{P}, C_{r}(s)$ and $D(s)$ respectively. Fig.5(b) depicts the implementation of the full nonlinear and robust IMC (NR-IMC) controller.

(a)

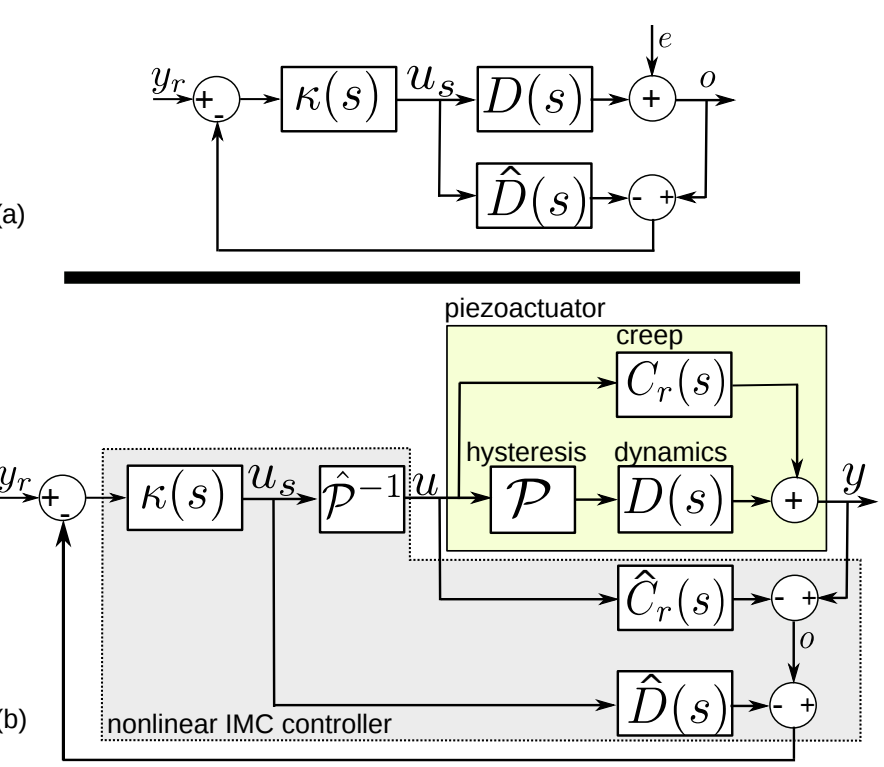

Fig. 5. (a) the IMC controller, and (b) the implemented nonlinear IMC controller.

The NR-IMC described in the previous subsection was implemented following the diagram in Fig. 5-b. The models $\hat{\mathcal{P}}, \hat{C}_{r}(s)$ and $\hat{D}(s)$ identified in section. II-C have been used for that. The gabarit $W(s)$ was derived using Eq. 13 a desired settling time of $t_{r}=15 \mathrm{~ms}$ which is well-suited to precise positioning tasks and characterization at the micro/nanoscale.

First a step input reference of $23 \mu \mathrm{m}$ was applied to the closed-loop. Fig.6(a) shows that the specified performance for the transient part is satisfied. The settling time is about $15 \mathrm{~ms}$ and the initial overshoot of $35 \%$ (see Fig.3(b)) is completely damped. Then, the harmonic response of the closed-loop was carried out. Fig.6(b) depicts the result which confirms the non-presence of resonance with the proposed RN-IMC controller. From the harmonic response, the $3 d B$ bandwidth is evaluated at $113 \mathrm{~Hz}$ which is useful in microrobotic based precise positioning tasks and in micro-scale characterization task [4]. 

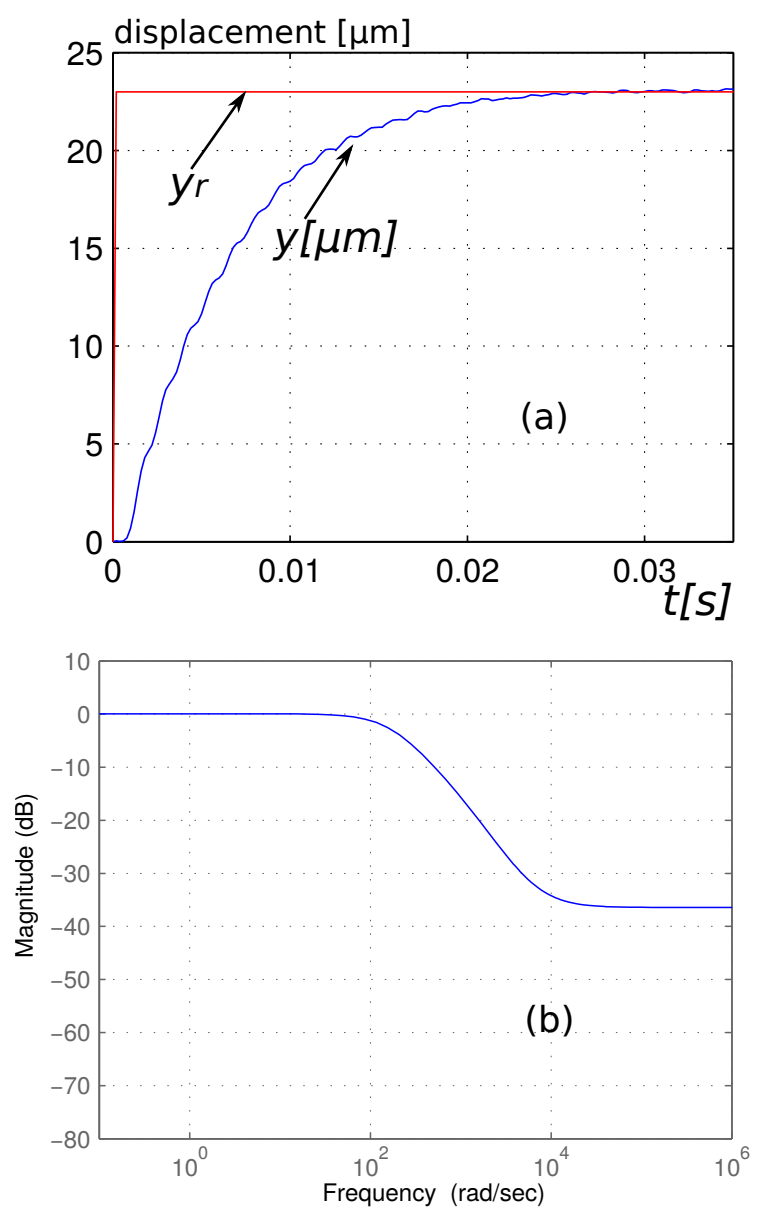

Fig. 6. Responses of the closed-loop control system: (a) step response, and (b) harmonic response.

\section{CONCLusions}

A creep-Hammerstein model that can characterize the hysteresis, creep, and linear dynamics of a piezocantilever actuator has been used to propose a nonlinear robust internal model control. The experimental results show that the proposed control system compensates for the hysteresis and creep nonlinearities and stabilizes the linear dynamics of the actuator. The future research will focus on: (i) internal model control for multi-axial piezoceramic stages, and (ii) exact mathematical formulation for the compensation errors of the proposed internal model control.

\section{ACKNOWLEDGMENT}

This work has been supported by the Labex ACTION project (contract ANR-11-LABX-0001-01).

\section{REFERENCES}

[1] F. Stefanski, B. Minorowicz, J. Persson, A. Plummer, and C. Bowen, "Non-linear control of a hydraulic piezo-valve using a generalised PrandtlIshlinskii hysteresis model," Mechanical Systems and Signal Processing, vol. 82, pp. 412-431, 2017.

[2] X. Liu, m. Kojima, Q. Shi, H. Wang, T. Sun, Y. Mae, Q. Huang, T. Arai, and T. Fukuda, "Non-contact transportation and rotation of micro objects by vibrating glass needle circularly under water," Proceedings of IEEE International Conference on Robotics and Automation, Singapore, pp. 5996-6001, 2017.
[3] J. Kim, S. Jeong, S. Han, and J. Lee, "Effects of different piezo-acting mechanism on two-stage fuel injection and CI combustion in a CRDi engine," Journal of Mechanical Science and Technology, vol. 30, pp. 5727-5737, 2016.

[4] M. Rakotondrabe, "Smart materials-based actuators at the micro/nanoscale: characterization, control and applications," Springer - Verlag, New York, ISBN 978-1-4614-6683-3, 2013.

[5] M. Rakotondrabe, "Piezoelectric systems for precise and high dynamic positioning: design, modeling, estimation and control," HDR, University of Franche-Comt / FEMTO-ST, November 10, 2014.

[6] L. Ryba, J. Dokoupil, A. Voda, and G. Besanon, "Adaptive hysteresis compensation on an experimental nanopositioning platform," International Journal of Control, vol. 90, pp. 765-778, 2017.

[7] M. Al Janaideh, M. Rakotondrabe, and X. Tan, "Guest editorial focused section on Hysteresis in smart mechatronic systems: modeling, identification, and control," IEEE/ASME Transactions on Mechatronics, vol. 21, pp. 1-3, 2016.

[8] V. Hassani, T. Tjahjowidodo, and T. Do, "A survey on hysteresis modeling, identification and control," Mechanical systems and signal processing, vol. 49, pp. 209-233, 2014.

[9] M. Al Janaideh, M. Rakotondrabe, I. Al-Darabsah, and O. Aljanaideh, "Internal model-based feedback control design for inversion-free feedforward rate-dependent hysteresis compensation of piezoelectric cantilever actuator," Control Engineering Practice, vol. 72, pp. 29-41, 2018.

[10] M. Rakotondrabe, 'Multivariable classical Prandtl-Ishlinskii hysteresis modeling and compensation and sensorless control of a nonlinear 2-dof piezoactuator', Springer Nonlinear Dynamics, DOI: 10.1007/s11071017-3466-5, March 2017.

[11] M. Al Janaideh, M. Rakotondrabe, and O. Aljanaideh, "Further results on hysteresis compensation of smart micro-positioning systems with the inverse Prandtl-Ishlinskii Compensator," IEEE Transactions on Control Systems Technology, vol. 24, pp. 428-439, 2016.

[12] O. Aljanaideh, D. Habineza, M. Rakotondrabe, and M. Al Janaideh, Experimental comparison of rate-dependent hysteresis models in characterizing and compensating hysteresis of piezoelectric tube actuators, Physica B, vol. 486, pp. 64-68, 2016.

[13] M. Rakotondrabe, O. Aljanaideh, and M. Al Janaideh, " $H_{\infty}$ control for a smart micro-positioning system with an analytical model for the output of the inverse compensation," Proceedings of American Control Conference, pp. 2643-2648, 2015.

[14] X. Tan and J. Baras, "Modeling and control of hysteresis in magnetostrictive actuators," Automatica, vol. 40, pp. 1469-1480, 2014.

[15] C. Visone and W. Zamboni, "Loop Orientation and Preisach Modeling in Hysteresis Systems" IEEE Transactions on Magnetics, vol. 51, pp. $1-4,2015$.

[16] A. Radouane, T. Ahmed-Ali, and F. Giri, "Parameter identification of Hammerstein systems with Bouc-Wen hysteresis input nonlinearity," Proceedings of European Control Conference, pp. 684-689, 2014.

[17] D. Habineza, M. Rakotondrabe, and Y. Le Gorrec, "BoucWen modeling and feedforward control of multivariable hysteresis in piezoelectric systems: application to a $3-\mathrm{DoF}$ piezotube scanner," IEEE Transactions on Control Systems Technology, vol. 23, pp. 1797-1806, 2015.

[18] B. Mokaberi and G. Requicha, "Compensation of scanner creep and hysteresis for AFM nanomanipulation," IEEE Transactions on Automation Science and Engineering, vol. 5, pp.197-208, 2008.

[19] M. Rakotondrabe, "Modeling and Compensation of Multivariable Creep in multi-DOF Piezoelectric Actuators," Proceedings of the International Conference on Robotics and Automation, pp. 4577-4581, Minnesota USA, 2012.

[20] D. Croft, G. Shed, and S. Devasia, "Creep, hysteresis and vibration compensation for piezoactuators: atomic force microscopy application," ASME Journal of Dynamic Systems, Measurement and Control, vol. 123, pp. 35-43, 2001.

[21] M. Al Janaideh, "About the output of the inverse compensation of the Prandtl-Ishlinskii model," Proceedings of the American Control Conference, Washington, DC, pp. 247-252, 2013.

[22] L. Ljung, System identification toolbox, The Matlab user's guide, 1988.

[23] R. Conant and W. Ashby, "Every good regulator of a system must be a model of that system," International Journal of Systems Science, vol. 1, pp. 89-97, 1970.

[24] K. Astrom, C. Hang, and B. Lim, "A new Smith predictor for controlling a process with an integrator and long dead-time," IEEE Transactions on Automatic Control, vol. 39, pp. 343-345, 1994. 